\title{
Calling into Question the Efficacy of Evidence-Based Medicine: Is It Always the Best Approach? Is That Really the Placebo Effect?
}

\author{
Yoshiro Fujii \\ Shin Kobe Dental Clinic, Kobe City, Japan \\ Email: shin-kobe-dentalclinic@s9.dion.ne.jp
}

Received 3 February 2015; accepted 27 March 2015; published 31 March 2015

Copyright (C) 2015 by author and Scientific Research Publishing Inc. This work is licensed under the Creative Commons Attribution International License (CC BY). http://creativecommons.org/licenses/by/4.0/

\begin{abstract}
The techniques of evidence-based medicine (EBM) are being frequently used recently. The generally accepted theory is that if a medicine is not absorbed by the body, it will have no effect. However, the author has noticed that even when substances, including medicines, are not absorbed by the body, they can have a marked effect. In some cases, just bringing the substance close to the body can have an effect; in such cases, the efficacy of EBM for estimating the therapeutic value of medicines may be debatable. Therefore, a more accurate evaluation method should be devised. The author proposes that another group be introduced into trials. This group would include subjects who were exposed to but did not have an opportunity to absorb the medicine being tested.
\end{abstract}

\section{Keywords}

Evidence-Based Medicine, Cohort Study, Randomized Controlled Trial, Electromagnetic Waves

\section{Introduction}

The techniques of evidence-based medicine (EBM) are being frequently used recently for evaluating the efficacy of medical technology and medicines [1]-[3]. The quality of the evaluation can range from meta-analysis and systematic reviews of double-blind, placebo-controlled clinical trials at the top end to conventional wisdom at the bottom (Table 1). The top three forms of evidence are as follows: 1) obtained from at least one properly designed randomized controlled trial; 2) obtained from well-designed controlled trials without randomization; and 3) obtained from well-designed cohort or case-control analytic studies, preferably from more than one center or 
Table 1. Levels of evidence for therapeutic studies*

\begin{tabular}{cl}
\hline Level & \multicolumn{1}{c}{ Type of evidence } \\
\hline 1A & Systematic review (with homogeneity) of RCTs \\
1B & Individual RCT (with narrow confidence intervals) \\
1C & All or none study \\
2A & Systematic review (with homogeneity) of cohort studies \\
2B & Individual cohort study (including low quality RCT, e.g., $<80 \%$ follow-up) \\
2C & "Outcomes” research; Ecological studies \\
3A & Systematic review (with homogeneity) of case-control studies \\
3B & Individual case-control study \\
4 & Case series (and poor quality cohort and case-control study \\
5 & Expert opinion without explicit critical appraisal or based on physiology bench research or "first principles" \\
\hline
\end{tabular}

*From the Centre for Evidence-based Medicine, http://www.cebm.net (last checked: 8 Jan. 2015).

research group. These methods are based on the concept that medicines have no efficacy unless they are directly absorbed by the body. However, there is a report that the Bi-Digital O-Ring Test [4] can be used to quickly detect the effect (positive or negative) of foods and nutritional supplements for an individual without taking them. This test suggests that even if these aren't taken in, a body reacts [5]. The purpose of this report is to call into question the reliability of EBM using evidence collected from two subjects who showed physical changes when exposed to substances that were not absorbed by their bodies.

\section{Case Reports}

\subsection{Case 1}

Subject and methods

The subject was a woman in her 40s, who had previously suffered a herniated lumbar disc about five years before the experiment which will be described below. The orthopedic doctor in charge suggested an operation to her at that time. However, her symptoms improved with occlusal treatment and drug treatment. Although, she did not feel any pain in her daily life, she felt pain in her back when the straight leg raising (SLR) test was performed on her right leg (Figure 1). Before the test, the subject was blindfolded to eliminate any psychological influences. A package of traditional Chinese medicine (Figure 2) which was judged to be effective by the Bi-Digital O-Ring Test [4] [5], was then slightly cut open and placed near the right side of the subject's back. Next, the SLR test was repeated and the subject's physical condition was assessed. In a second test, the unblindfold subject's finger floor distance (FFD) was measured. First, the subject was asked to bend forward at the waist, and her FFD was assessed. Second, the same package of Chinese medicine was placed on the floor just in front of her. Further, the subject's FFD was reassessed.

Results

The subject exhibited physical changes during both tests. When the medicine was placed near her back during the first test, she no longer reported back pain when the SLR test was performed on her right leg. Moreover, her joint mobility also showed improvement (Figure 3). When the subject's initial FFD was measured, it was approximately $15 \mathrm{~cm}$ (Figure 4). However, once the medicine was placed in front of her, she was able to touch the floor (Figure 5). When the medicine was removed while the subject was touching the floor, her FFD returned to $15 \mathrm{~cm}$. Further, when a double amount of medicine was placed in front of her, she was still able to touch the floor; however, when the amount was tripled, her FFD returned to $15 \mathrm{~cm}$. At the time of submitting, her back condition was still stable. The author suggested that she tape the medicine to her back if her lumbago becomes worse.

In order to watch the actual experiment described in this case, please visit You Tube movie:

Criticism of EBM (Evidence-based Medicine)

https://www.youtube.com/watch?v=SscNNIfgfaU

(last checked: 8 Jan 2015) 


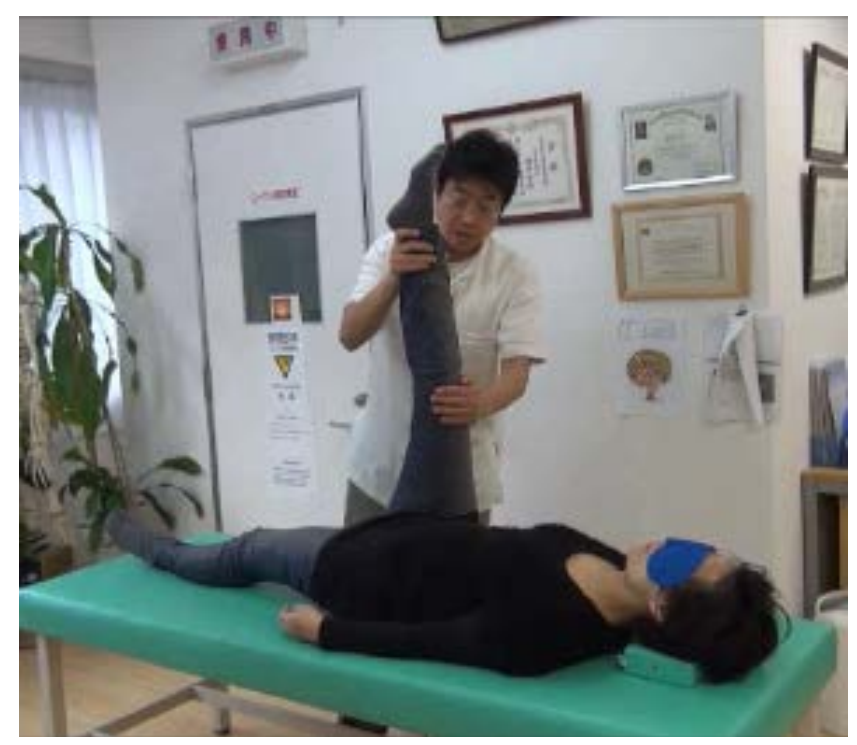

Figure 1. The blindfold subject had complaining of pain during right straight leg raising (SLR) test at her right back.

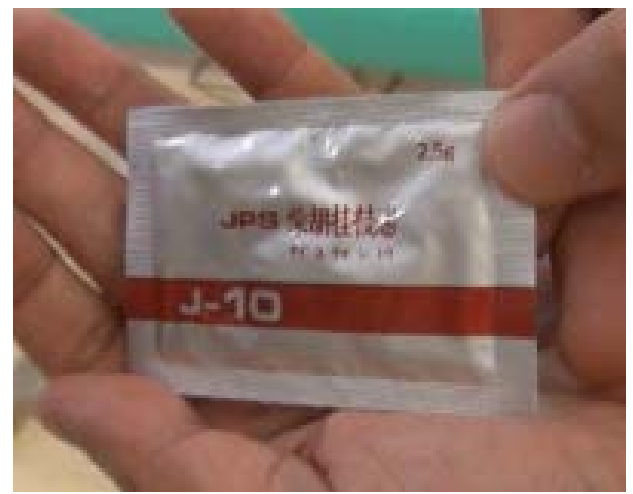

Figure 2. Chinese medicine extract judged to be effective for the subject's back pain.

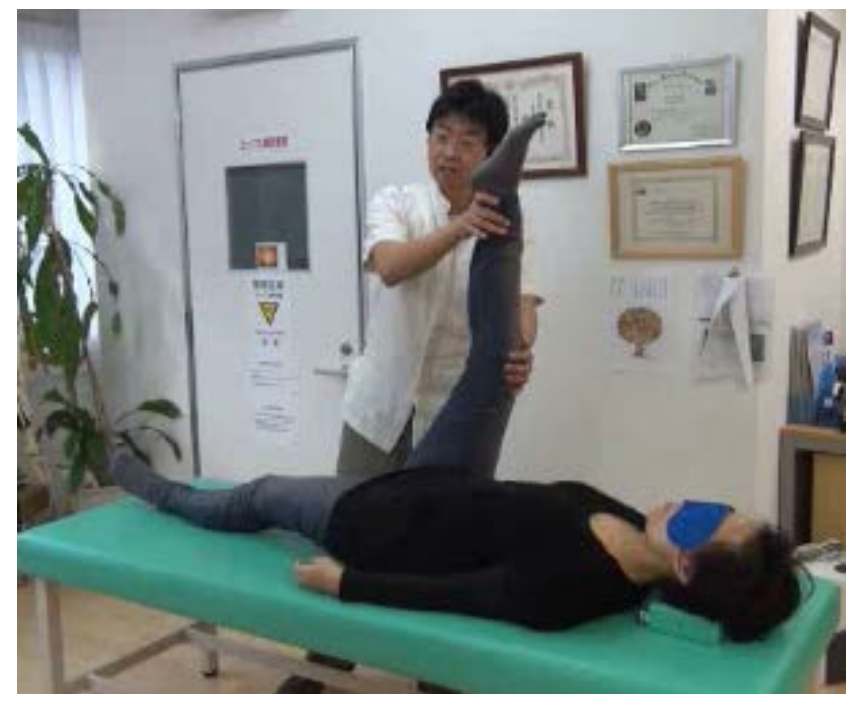

Figure 3. When the author made a small cut in a bag of the Chinese medicine extract indicated on Figure 2 and put it in the place where the author approached a subject (the medication was not touching the subject), the pain at her back disappeared and also increased a joint mobility at the time of SLR. 


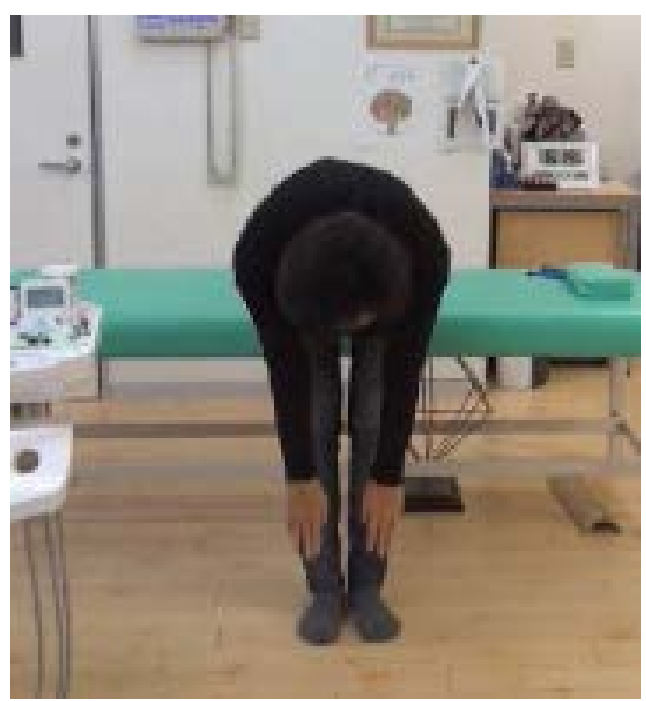

Figure 4. When the subject bent her body forward, the finger floor distance (FFD) was approximately $15 \mathrm{~cm}$.



Figure 5. When the Chinese medication extract (indicated in Figure 2) was located in front of the subject (arrow), her fingertip touched the floor. When the amount of extract was doubled, her fingertip was located at same position, the forward bending amount did not change; however, when the extract was tripled, her FFD returned to $15 \mathrm{~cm}$.

\subsection{Case 2}

Subject, method, and result

The subject was a woman in her 60s. She had a lower full denture. It was made of resin (Polymethyl methacrylate) and alloy (Au 87\%, Pt 11\%, and the other). It was judged to be effective by the Bi-Digital O-Ring Test [4] [5]. When she bent forward while blindfolded without her denture, her FFD was about $3 \mathrm{~cm}$. When the denture was secretly placed near her feet, she was able to touch the floor with her fingertips. When the denture was removed, she was unable to keep her fingertips on the floor because of the tension of her legs. When the denture was placed in her mouth, she could bend more easily than when the denture was placed near her feet.

\section{Discussion}

The techniques of EBM are frequently being used recently [1]-[3]. This study reveals that even when substances, including medicines, do not enter the body, they can have a marked effect on joint mobility. This appears to be true even when the substances are simply in close proximity to the body, indicating that medicine can influence 
a patient's condition in three ways: 1) the medicine can be absorbed by the body; 2) it can have an effect when in close proximity to the body; and 3) it can cause a placebo effect. The author suggests that the first mechanism be called the direct effect and the second be called the indirect effect of the medicine. Therefore, the outcome of taking any medicine will be the sum of the direct and indirect effects of the medicine on the patient. Modern Western medicine, including EBM, ignores the indirect effect. The indirect effect can have serious implications for the accuracy of the testing of new drugs. In the case of cohort studies, which are said to provide relatively high levels of evidence, subjects are divided into two groups. One group comprises those who take the medicine, and the other group comprises those who do not. The indirect effect could have unnoticed effects on such tests. For example, in the control group, some members may have medicine in their pockets. Despite the medicine being present in the pockets, real physical effects may be noticed on the individuals; such effects will not be classified as authentic by the investigators. These effects will be defined as placebo effects. When comparing effects, subjects should be classified into three groups: 1) those who took medicine; 2) those who had medicine in close proximity but did not take it; and 3) those from whom medicine is kept away and not taken. In randomized controlled trials (RCTs), a placebo is administered to some subjects, while the target medication is administered to others. The results are then compared. A placebo should have no positive or negative effects on subjects. However, if the placebo exerts a strong indirect effect on the body, the evaluation of the test medication could then be inaccurate. In summary, drug effectiveness measurements based on EBM cannot be trusted because of the indirect effect, which has not been accounted for in EBM to date. Moreover, according to Western pharmacological theory, the larger the dose of medicine taken, the greater the effect of the medicine (dose-response relationship) [6]. However, this study suggests that the dose-response relationship does not consider the indirect effect of a medicine. It shows that at some point, increasing the dose facilitates reaching the maximum level of indirect effect, following which, the efficacy of the medicine declines as the dose is increased. The mechanism underlying the indirect effect remains unclear; therefore, this decline in effectiveness cannot be presently explained. Further research is required. One hypothesis is that the indirect effect is the result of the unique electromagnetic waves emitted by every substance, including medicines. An indication of this comes from the packaging of the medicine used in this experiment. When the package, made of aluminum, was unopened, the patient was shielded from the medicine and little indirect effect was observed. However, when the package was cut open, the indirect effect became stronger. The author confirmed that even if the opening in the package is sealed using Scotch tape, to prevent exposing the subject to the volatile components from the medicine or from the aromatic effect of the medicine, the same result occurs. The author believes that the mechanism which is causing the indirect effects on the subject is something like electromagnetic waves. That is, it can be blocked by aluminum foil, such as that used in the package of the Chinese medicine, but it can pass through plastics, such as the Scotch tape. The author has suggested that the electromagnetic waves emitted by cell phones and PCs may cause scoliosis, balance dysregulation, and joint mobility disorder [7]-[13]. The author has also reported that such issues decreased when aluminum foil was placed between an electromagnetic wave-emitting electronic device and the subject [8] [13]. To date, according to a dose-response relationship, it is said that the larger the dose of medicine taken, the greater the effect of the medicine [6]. However, based on these results, when a medication is judged to be ineffective, a decrease in the dosage should also be considered.

If there are severe side effects, the indirect effect could be much better than the direct effect. For example, medicine could be placed in the patient's pocket. The patient would receive the benefits of the indirect effect of the medicine without the side effects of the direct effect. In such a case, the efficacy of EBM for estimating the therapeutic value of medicines may be debatable. In another case, the placebo used by the control group may have noticeable indirect effects on the body. Moreover, in such a case, the results of past randomized controlled trials and cohort or case-control studies may be inaccurate. Therefore, an evaluation method more accurate than EBM is desired. I propose that a third group be introduced into trials. This group should include subjects who were exposed to but who did not ingest the test medicine, eliminating the inaccuracies that are present in the current testing methods. Even if the indirect effect has unwanted side effects, they can be quickly stopped by removing the medicine. This phenomenon can be easily confirmed through reproducing the authors experiment. Each time a different metal is placed on the floor in front of a subject, their observed FFD will change. This report has presented two cases that clear show the indirect effects of dental materials and medicines on the flexibility of two subjects. Unfortunately, two cases are not a large enough group to be statistically valid. The author hopes to collect further evidence in the future in order to show that these results are wide-spread and quantifiable. 


\section{Conclusion}

Three action mechanisms of medicines are believed to exist: the direct, the indirect, and placebo effects. The indirect effect is ignored in modern Western medicine. If there is an effect, which is because of the indirect effect, it is classified as the placebo effect. However, the cases presented in this paper reveal that even when substances, including medicine, are not absorbed by the body, they can have a noticeable effect on joint mobility. In such a case, the results of past randomized controlled trials and cohort or case-control studies may be inaccurate. Therefore, an evaluation method more accurate than EBM should be devised. The author proposes that a third group be introduced into trials. This group would include subjects who were exposed to but who did not ingest the test medicine, thereby eliminating the inaccuracies present in the present testing methods.

\section{References}

[1] Guyatt, G.H., Sackett, D.L. and Cook, D.J. (1994) Users' Guides to the Medical Literature, II: How to Use an Article about Therapy or Prevention, Part B: What Were the Results and Will They Help Me in Caring for My Patients? JAMA, 271, 59-63. http://dx.doi.org/10.1001/jama.1994.03510250075039

[2] Jaeschke, R.Z., Guyatt, G.H. and Sackett, D.L. (1994) Users' Guides to the Medical Literature, III: How to Use an Article about a Diagnostic Test, Part B: What Are the Results and Will They Help Me in Caring for My Patients? JAMA, 271, 703-707. http://dx.doi.org/10.1001/jama.1994.03510330081039

[3] Guyatt, G.H., Haynes, R.B., Jaeschke, R.Z., Cook, D.J., Green, L., Naylor, C.D., Wilson, M.C. and Richardson, W.S. (2000) Users' Guides to the Medical Literature: XXV. Evidence-Based Medicine: Principles for Applying the Users' Guides to Patient Care. Evidence-Based Medicine Working Group. JAMA, 284, 1290-1296. http://dx.doi.org/10.1001/jama.284.10.1290

[4] Omura, Y. (1993) Bi-Digital O-Ring Test for Imaging and Diagnosis of Internal Organs of a Patient. US Patent 5188107. http://academic.reed.edu/economics/parker/f11/354/pat/o-ring.pdf

[5] http://bdort.org/ (last checked :27.Mar.2015).

[6] Altshuler B. (1981) Modeling of Dose-Response Relationships. Environmental Health Perspectives, 42, $23-27$. http://dx.doi.org/10.1289/ehp.814223

[7] Fujii, Y. (2012) Do Dental Implants Cause Scoliosis? Case Report. Personalized Medicine Universe, 1, 79-80. http://dx.doi.org/10.1016/j.pmu.2012.05.012

[8] Fujii, Y. (2014) Gold Alloy Dental Inlay for Preventing Involuntary Body Movements Caused by Electromagnetic Waves Emitted by a Cell Phone. Open Journal of Antennas and Propagation, 2, 37-43. http://dx.doi.org/10.4236/ojapr.2014.24005

[9] Fujii, Y. (2014) Sense of Balance Disorder Caused by Electromagnetic Waves Collected by a Dental Implant. Acupuncture and Electro-Therapeutics Research, 39, 379.

[10] Fujii, Y. (2014) Sensation of Balance Dysregulation Caused/Aggravated by a Collection of Electromagnetic Waves in a Dental Implant. Open Journal of Antennas and Propagation, 2, 29-35. http://dx.doi.org/10.4236/ojapr.2014.23004

[11] Fujii, Y. (2007) The Dental Treatment That Used Environment of Electromagnetic Wave. Acupuncture and ElectroTherapeutics Research, 32, 291.

[12] Fujii, Y. (2009) The Consideration of the Electromagnetic Wave in Dental Material Substitution. Acupuncture and Electro-Therapeutics Research, 34, 89.

[13] Fujii, Y. (2015) Dental Treatment for Dizziness and Joint Mobility Disorder Caused by Harmful Electromagnetic Waves. Open Journal of Antennas and Propagation, 3, 1-7. http://dx.doi.org/10.4236/ojapr.2015.31001 